\title{
COVID-19 Pneumonia: An Emerging Cause of Syndrome of Inappropriate Antidiuretic Hormone
}

\author{
Muhammad Mubbashir Sheikh ${ }^{1}$, Ejaz Ahmad ${ }^{2,3}$, Hafiz Muhammad Jeelani ${ }^{4}$, Adeel Riaz ${ }^{5}$, Ahmad \\ Muneeb 6 \\ 1. Oncology, Northwestern University Feinberg School of Medicine, Chicago, USA 2. Internal Medicine, Mayo Hospital, \\ Lahore, PAK 3. Internal Medicine, Nishtar Hospital, Multan, PAK 4. Internal Medicine, Rosalind Franklin University of \\ Medicine and Science, McHenry, USA 5. Anesthesiology and Critical Care, District Headquarter Hospital, Sahiwal, PAK \\ 6. Internal Medicine, Allied Hospital / Faisalabad Medical University, Faisalabad, PAK
}

Corresponding author: Muhammad Mubbashir Sheikh, mubbashirsheikh260@gmail.com

\begin{abstract}
Syndrome of inappropriate antidiuretic hormone (SIADH) is the leading cause of hyponatremia. We, herein, report a case of a patient with coronavirus disease-2019 (COVID-19) who developed sudden exertional dyspnea and hypoxia and was found to be hyponatremic. A diagnosis of SIADH was made due to COVID-19 pneumonia. The patient was managed conservatively with a significant improvement during the course of hospitalization and on follow-up.
\end{abstract}

Categories: Medical Education, Nephrology, Pulmonology

Keywords: siadh, hyponatremia, covid-19

\section{Introduction}

Since December 2019, the outbreak of coronavirus disease 2019 (COVID-19) from Wuhan, China, has not only caused global social and economic determinants but also profoundly impacted the current context of scientific research. The disease has been reported to involve many organs, particularly, lungs, kidneys, and is linked to a myriad of complications resulting in high mortality across the globe [1]. As of May 28, 2020, more than 6,000,000 confirmed cases and more than 350,000 deaths have been documented worldwide [2]. The symptoms of COVID-19 are mostly dependent on the age and the immunity of the patients [3]. Most common symptoms encountered during clinical practice include fever (88.7\%), cough (57.7\%), dyspnea (45.6\%), and diarrhea (3.8\%) [4]. Recently, hyponatremia has been reported as a common electrolyte disorder in COVID-19 patients [5-6]. Similar to our patient, the limited data have shown COVID-19 pneumonia as an underlying cause of syndrome of inappropriate antidiuretic hormone secretion (SIADH) induced hyponatremia [7-8]. SIADH is a diagnosis of exclusion characterized by dilutional hyponatremia in the absence of any identifiable renal pathology, diuretics intake, or any other no-osmotic stimulating factors for antidiuretic hormone (ADH) production [9-10]. Our case report adds to the ongoing research that proposes the significance of recognizing the fundamental etiology for hyponatremia in patients with COVID-19. As treatment can vary for hyponatremia in different underlying conditions, it is of paramount importance to have the proper management to prevent consequential complications.

Received 06/09/2020 Review began $06 / 20 / 2020$ Published 06/26/2020

\section{(c) Copyright 2020}

Sheikh et al. This is an open access article distributed under the terms of the Creative Commons Attribution License CC-BY 4.0., which permits unrestricted use, distribution, and reproduction in any medium, provided the original author and source are credited.

\section{Case Presentation}

A 37-year-old male with no significant past medical history presented to the ED with complaints of fever, dry cough, malaise for three days, and progressively worsening dyspnea for one day. No additional symptoms were reported by the patient. Social history was negative for smoking, alcohol, or illicit drugs. Clinically, the patient was alert and oriented to time, place, and person. Physical examination revealed a temperature of $101^{\circ} \mathrm{F}$, normotensive, respiratory rate (RR) of 28 breaths per minute, and heart rate (HR) of 115 beats per minute. His oxygen saturations were $88 \%$ on ambient air. Further, a focused respiratory exam revealed reduced air entry and bilateral scattered wheezing on auscultation. The rest of the pertinent physical examination was negative for pedal edema, hepatomegaly, jugular venous distension (JVD), or any heart murmur.

Laboratory investigations at the time of admission revealed significant low serum sodium (Na) level of 118 meq/L (normal range: 135-145 meq/L) with a serum osmolality of 239 mOsm $/ \mathrm{kg}$ (normal range: 285-294 $\mathrm{mOsm} / \mathrm{kg}$ ), a white blood cell (WBC) count of 7.92 cells $/ \mathrm{mm} 3$ (normal range: $4-11$ cells $/ \mathrm{mm} 3$ ) with $86 \%$ neutrophils (normal range: $40 \%-75 \%$ ) and 10\% lymphocytes (normal range: $18 \%-42 \%$ ). Serum potassium, creatinine, calcium and magnesium levels, liver function tests, and coagulation profile including prothrombin time (PT), activated partial thromboplastin time (aPTT) were within the normal range. Chest Xray (CXR) showed a pneumonitis patch in the peripheral region of the right mid zone and bilateral alveolar infiltrates, highly suggestive of COVID-19 pneumonia (Figure 1). Nasopharyngeal swab via real-time reverse transcriptase-polymerase chain reaction analysis (RT-PCR) confirmed COVID-19. Further, the workup for euvolemic hyponatremia revealed urine osmolality of $338 \mathrm{mOsm} / \mathrm{kg}$ (normal range: 50-1200 mOsm/ $\mathrm{kg}$ ) and urine spot sodium $45 \mathrm{mmol} / \mathrm{L}$ (normal range: $54-190 \mathrm{mmol} / \mathrm{L}$ ). Additionally, the normal range levels of 


\section{Cureus}

thyroid-stimulating hormone and cortisol exclude adrenal pathology as a cause of hyponatremia. A diagnosis of COVID-19 pneumonia and SIADH was made.

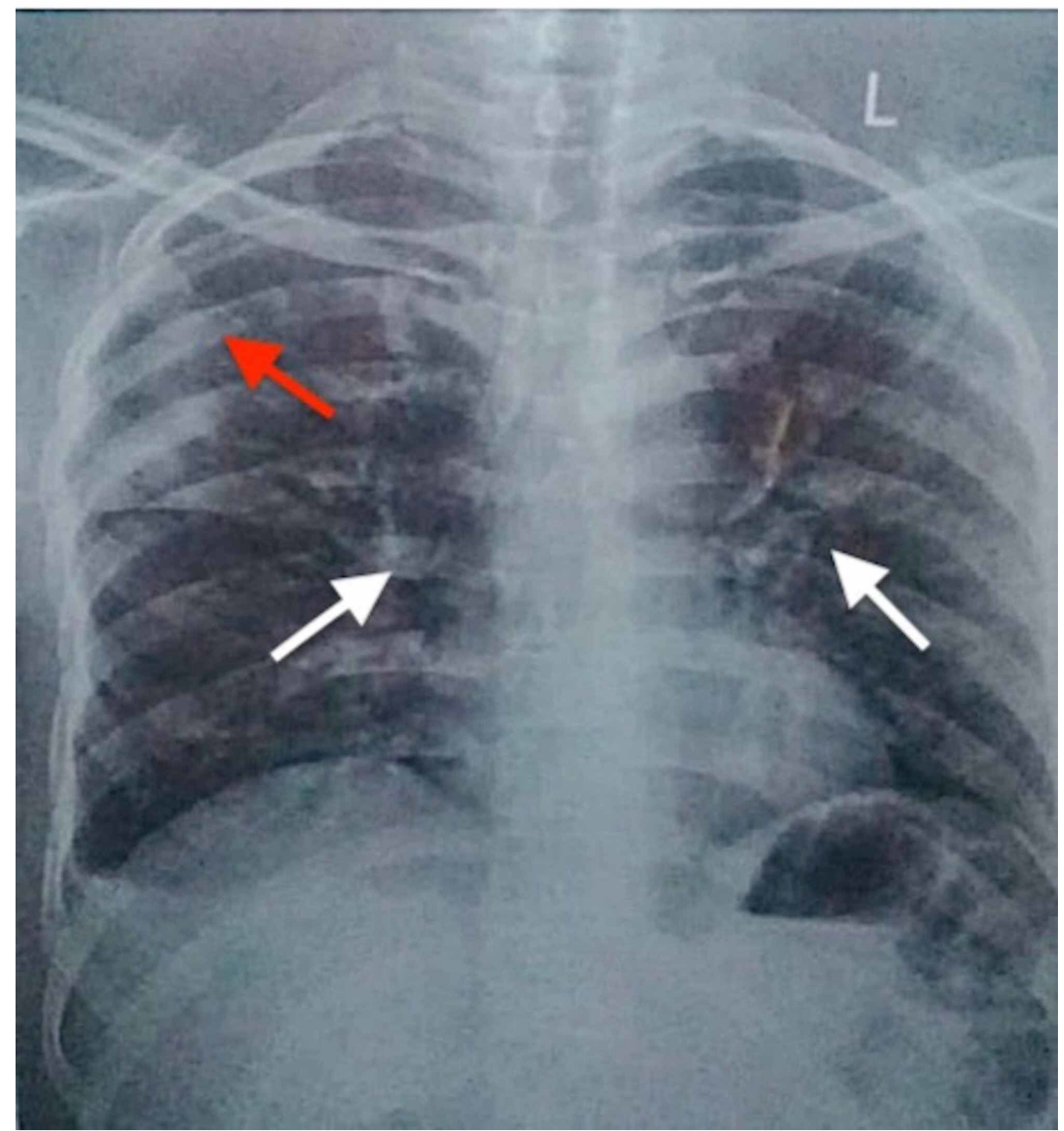

\section{FIGURE 1: Chest radiograph showing pneumonitis patch in the peripheral region of right mid zone (red arrow) and bilateral alveolar infiltrates (white arrows).}

Initially, the patient was managed with appropriate oxygenation, intravenous moxifloxacin, and acetaminophen on the lines of community-acquired pneumonia.

Fluid restriction of $1 \mathrm{~L} /$ day $(1000 \mathrm{~mL} /$ day $)$ was done. After 12 hours of confirmed suspicion of novel coronavirus i.e., severe acute respiratory syndrome coronavirus (SARS-Cov-2), the patient was transitioned to COVID-19 designated isolation ward. Serum sodium levels repeated at 24, 48, and then 72 hours revealed levels of 123, 129, and $136 \mathrm{mEq} / \mathrm{L}$, respectively. Concerning COVID-19 pneumonia, moxifloxacin was stopped, azithromycin $500 \mathrm{mg}$ once daily for five days, zinc sulfate $50 \mathrm{mg}$, and vitamin C $500 \mathrm{mg}$ (ascorbic acid) for 14 days each were administered. During hospitalization, a marked improvement in respiratory status with decreased oxygen requirement was observed. At two weeks of follow-up, labs (WBC count of 6.18 with $65 \%$ neutrophils and $18 \%$ lymphocytes, and serum Na level of $139 \mathrm{mEq} / \mathrm{L}$, respectively) and CXR (accentuated bronchovascular markings observed bilaterally with no acute exudative lesion, lymphadenopathy, or pleural fluid) depicted the improved baseline health status of the patient.

\section{Discussion}

Hyponatremia is a frequently encountered electrolyte disorder in hospitalized patients and a predictor of overall mortality and morbidity [11]. SIADH has been identified as a predominant cause of hyponatremia in up to $50 \%$ of cases [12]. Table 1 lists the diagnostic criteria for SIADH [9-10]. 


\section{Cureus}

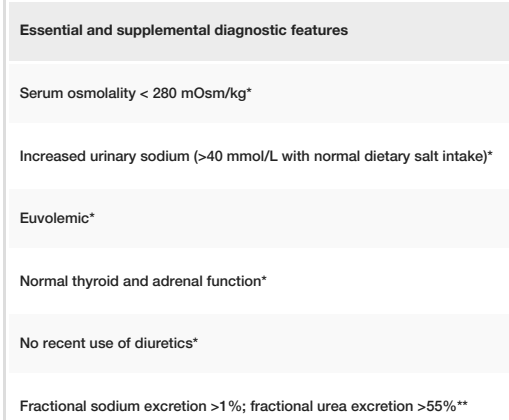

Elevated plasma ADH levels, despite the presence of hypotonicity and clinical euvolemia*

\section{TABLE 1: Diagnostic criteria of SIADH.}

SIADH: syndrome of inappropriate antidiuretic hormone; ADH: antidiuretic hormone

*Essential diagnostic features; ${ }^{\star \star}$ Supplemental diagnostic features

Common lung pathologies such as pulmonary malignancy, severe obstructive lung disease, acute respiratory failure, and pneumonia are the prevalent disorders that can lead to SIADH [9]. Among viral infections, influenza has been identified as an underlying cause of SIADH [13-14]. In recent times, COVID-19 pneumonia has emerged as a causative factor for SIADH. The pathogenesis for the development of SIADH in COVID-19 pneumonia per preliminary reports is due to the production of certain proinflammatory cytokines, particularly, IL-6. These cytokines increase the ADH production via twofold mechanisms: firstly, by direct stimulation of nonosmotic release of ADH, and secondly by the direct insult of alveolar basement membrane resulting in activation of hypoxic pulmonary vasoconstriction pathway that can lead to increase ADH production [15-18]. Thus, suggesting endogenous nonosmotic ADH production. However, further studies are needed to extrapolate this mechanism.

The effective management of SIADH depends on the identification of underlying etiologies, determination of serum sodium (Na) levels, and urine osmolality. Table 2 lists the classification of hyponatremia with common underlying conditions [12]. Close monitoring of Na levels and urine osmolality will dictate the rate of fluid resuscitation and will also help to avoid exacerbation of respiratory distress especially in patients with pulmonary infections or disorders [19]. In hyponatremia either acute ( $<48$ hours) or chronic ( $>48$ hours) fluid correction should be done cautiously to avoid the precipitous drop of $\mathrm{Na}$ levels of $>8 \mathrm{mEq} / \mathrm{L}$ over a period of 24 hours in order to prevent the risk of associated complications [20]. General treatment includes the administration of aggressive hypertonic saline (acute hyponatremia) and fluid restriction to less than water excretion in (chronic hyponatremia) or asymptomatic patients with SIADH [12]. Other options that can be utilized in SIADH are loop diuretics, vasopressin receptor antagonists, demeclocycline, and oral salt tablets [19-20]. Fluid restriction along with supportive management of COVID-19 was the mainstay to correct SIADH in our patient.

\begin{tabular}{|c|c|c|}
\hline Hypervolemia (UNa excretion - low, plasma renin - high) & Hypovolemia (UNa excretion - low or high, plasma renin - high) & Euvolemia (UNa excretion - low or high, plasma renin - low) \\
\hline Congestive heart failure ${ }^{*}$ & Bleeding* & $\mathrm{SIADH}^{* *}$ \\
\hline Cirrhosis ${ }^{*}$ & Diuretic use ${ }^{*}$ & Primary polydipsia* \\
\hline Nephrotic sync & rrhea $^{*}$ & Hypothyroidism \\
\hline & Adrenal insufficiency & \\
\hline
\end{tabular}

TABLE 2: Hyponatremia classification with common underlying etiologies.

ADH: antidiuretic hormone; SIADH: syndrome of inappropriate antidiuretic hormone; UNa: urinary sodium

*Urine sodium excretion - low; **Urine sodium excretion - high 
Our case emphasizes the consideration of SIADH in hospitalized COVID-19 patients having hyponatremia. The correct identification of the underlying etiology in hyponatremia through detailed workup is critical for curtailing the length of hospital stay, inappropriate treatment, and debilitating morbidity in the current pandemic. In summary, COVID-19 pneumonia is an evolving cause of SIADH; however, future studies are warranted to explore this association.

\section{Additional Information \\ Disclosures}

Human subjects: Consent was obtained by all participants in this study. Conflicts of interest: In compliance with the ICMJE uniform disclosure form, all authors declare the following: Payment/services info: All authors have declared that no financial support was received from any organization for the submitted work. Financial relationships: All authors have declared that they have no financial relationships at present or within the previous three years with any organizations that might have an interest in the submitted work. Other relationships: All authors have declared that there are no other relationships or activities that could appear to have influenced the submitted work.

\section{References}

1. Lu H, Stratton CW, Tang YW: Outbreak of pneumonia of unknown etiology in Wuhan China: the mystery and the miracle. J Med Virol . 2020, 92:401-402. 10.1002/jmv.25678

2. COVID-19 Dashboard by the Center for Systems Science and Engineering (CSSE) at Johns Hopkins University (JHU). (2020). Accessed: May 28, 2020: https://coronavirus.jhu.edu/map.html.

3. Wang W, Tang J, Wei F: Updated understanding of the outbreak of 2019 novel coronavirus (2019-nCoV) in Wuhan, China. J Med Virol. 2020, 92:441-447. 10.1002/jmv.25689

4. Chen N, Zhou M, Dong X, et al.: Epidemiological and clinical characteristics of 99 cases of 2019 novel coronavirus pneumonia in Wuhan, China: a descriptive study. Lancet. 2020, 395:507-513. 10.1016/S01406736(20)30211-7

5. Hong XW, Chi ZP, Liu GY, et al.: Analysis of early renal injury in COVID-19 and diagnostic value of multiindex combined detection (Preprint). MedRxiv. 2020, 10.1101/2020.03.07.20032599

6. Aggarwal S, Garcia-Telles N, Aggarwal G, Lavie C, Lippi G, Henry BM: Clinical features, laboratory characteristics, and outcomes of patients hospitalized with coronavirus disease 2019 (COVID-19): early report from the United States. Diagnosis (Berl). 2020, 7:91-96. 10.1515/dx-2020-0046

7. Ravioli S, Niebuhr N, Ruchti C, Pluess E, Stoeckli T, Lindner G: The syndrome of inappropriate antidiuresis in COVID-19 pneumonia: report of two cases. Clin Kidney J. 2020, sfaa080. 10.1093/ckj/sfaa080

8. Yousaf Z, Al-Shokri SD, Al-Soub H, Mohamed MFH: COVID-19-associated SIADH: a clue in the times of pandemic!. Am J Physiol Endocrinol Metab. 2020, 318:E882-E885. 10.1152/ajpendo.00178.2020

9. Ellison DH, Berl T: Clinical practice. The syndrome of inappropriate antidiuresis . N Engl J Med. 2007, 356:2064-2072. 10.1056/NEJMcp066837

10. Schwartz WB, Bennet W, Curelop S, Barter FC: A syndrome of renal sodium loss and hyponatremia probably resulting from inappropriate secretion of antidiuretic hormone. Am J Med. 1957, 23:529-542. 10.1016/00029343(57)90224-3

11. Corona G, Giuliani C, Parenti G, et al.: Moderate hyponatremia is associated with increased risk of mortality: evidence from a meta-analysis. PLoS One. 2013, 8:e80451. 10.1371/journal.pone.0080451

12. Esposito P, Piotti G, Bianzina S, Malul Y, Dal Canton A: The syndrome of inappropriate antidiuresis: pathophysiology, clinical management and new therapeutic options. Nephron Clin Pract. 2011, 119:c62-c73. $10.1159 / 000324653$

13. Pathak R, Khanal A, Poudel DR, Karmacharya P: Down with the flu: hyponatremia in a patient with influenza. N Am J Med Sci. 2015, 7:227-228. 10.4103/1947-2714.157488

14. Gaglani B, Gupta S, Chavez O, Libardo R: Influenza as a cause of SIADH related hyponatremia: a case report . J Clin Diagn Res. 2017, 11:OD10-OD11. 10.7860/JCDR/2017/25785.9797

15. Mehta P, McAuley DF, Brown M, et al.: COVID-19: consider cytokine storm syndromes and immunosuppression. Lancet. 2020, 395:1033-1034. 10.1016/S0140-6736(20)30628-0

16. Park SJ, Shin JI: Inflammation and hyponatremia: an underrecognized condition?. Korean J Pediatr. 2013, 56:519-522. 10.3345/kjp.2013.56.12.519

17. Qin C, Zhou L, Hu Z, et al.: Dysregulation of immune response in patients with COVID-19 in Wuhan, China . Clin Infect Dis. 2020, 10.1093/cid/ciaa248

18. Mastorakos G, Weber IS, Magiakou MA, Gunn H, Chrousos GP: Hypothalamic-pituitary-adrenal axis activation and stimulation of systemic vasopressin secretion by recombinant interleukin- 6 in humans: potential implications for the syndrome of inappropriate vasopressin secretion. J Clin Endocrinol Metab. 1994, 79:934-939. 10.1210/jcem.79.4.7962300

19. Pillai BP, Unnikrishnan AG, Pavithran PV: Syndrome of inappropriate antidiuretic hormone secretion: revisiting a classical endocrine disorder. Indian J Endocrinol Metab. 2011, 15:S208-S215. 10.4103/22308210.84870

20. Sahay M, Sahay R: Hyponatremia: a practical approach. Indian J Endocrinol Metab. 2014, 18:760-771. $10.4103 / 2230-8210.141320$ 